AperTO - Archivio Istituzionale Open Access dell'Università di Torino

\title{
Co-operation in Social Dilemmas through Position Uncertainty
}

\section{This is a pre print version of the following article:}

Original Citation:

\section{Availability:}

This version is available http://hdl.handle.net/2318/1638538

since 2019-07-22T10:23:40Z

Published version:

DOI:10.1111/ecoj.12636

Terms of use:

Open Access

Anyone can freely access the full text of works made available as "Open Access". Works made available under a Creative Commons license can be used according to the terms and conditions of said license. Use of all other works requires consent of the right holder (author or publisher) if not exempted from copyright protection by the applicable law. 


\title{
Cooperation in Social Dilemmas through Position
}

\author{
Uncertainty*
}

\author{
Andrea Gallice and Ignacio Monzón
}

July 23, 2018

Short Title: Cooperation through Position Uncertainty

\begin{abstract}
We present a natural environment that sustains full cooperation in one-shot social dilemmas among a finite number of self-interested agents. Players sequentially decide whether to contribute to a public good. They do not know their position in the sequence, but observe the actions of some predecessors. Since agents realise that their own action may be observed, they have an incentive to contribute in order to induce potential successors to also do so. Full contribution can then emerge in equilibrium.

The same environment leads to full cooperation in the prisoners' dilemma.

${ }^{*}$ We thank Ben Cowan, Laura Doval, Juan Escobar, Dino Gerardi, Paolo Ghirardato, Edoardo Grillo, Toomas Hinnosaar, Johannes Hörner, Giorgio Martini, Bill Sandholm, and Alex Tetenov for valuable suggestions. We also thank seminar participants at the North American Summer Meeting of the Econometric Society (St. Louis), Conference on Economic Design (York), SAET Conference (Faro), Public Economic Theory Conference (Paris), EUI Alumni Workshop (Florence), International Conference on Game Theory (Stony Brook), European Meeting of the Econometric Society (Lisbon), Latin American Meeting of the Econometric Society (Buenos Aires), and Markets with Informational Asymmetries Workshop (Torino). Corresponding Author: Ignacio Monzón. Piazza Vincenzo Arbarello, 8, 10122 Torino, Italy (ignacio@carloalberto.org).
\end{abstract}


JEL Classification: C72, D82, H41.

Keywords: Social Dilemmas; Public Goods; Observational Learning; Position Uncertainty.

In social dilemmas, individual incentives and collective interests are at odds. Whenever cooperation is costly, the possibility to free ride on the effort of others can hinder the achievement of socially optimal outcomes. Cooperation between self-interested agents typically requires strategic interactions repeated over an infinite horizon. Alternatively, it can emerge when agents have nonstandard preferences (for instance, if they experience warm-glow effects from cooperating) or are not fully rational.

In this paper we show how full cooperation can arise in a natural environment, even when the interaction is one-shot, the number of players is finite, and agents are selfinterested. We present our result in the context of a public good game. To fix ideas, consider several wealthy individuals who may contribute to a new project for the community. Each potential contributor obtains utility from the project, but would rather have others funding it.

We show that contribution by all agents can emerge when individuals make decisions sequentially, do not know their position in the sequence, and observe the decisions of some of their predecessors. Consider a strategy that prescribes contribution, unless defection is observed. If agents knew their position, then those placed early in the sequence would contribute if they could induce their successors to do the same. However, late players would rather free ride on the effort of early contributors. Contribution would thus unravel. Instead, an agent who does not know her position bases her decision on the 
average payoffs from all possible positions. She contributes to induce her potential successors to do the same.

We present a simple environment: a finite number of agents must choose whether to contribute to a public good. Agents choose sequentially but do not know their position in the sequence: they are equally likely to be placed in any position. Before choosing an action, each agent observes her immediate predecessors' decisions. After all $n$ players have made their decisions, the total amount invested is multiplied by the return from contributions parameter $r$, and then equally shared among all agents. This multiplication factor satisfies $1<r<n$, so although it is socially optimal that all agents contribute, each agent would prefer not to contribute herself.

In our main result we show that full contribution can occur in equilibrium. Proposition 1 characterises the maximum level of contribution that can be achieved as a function of the return from contributions $r$. If $r$ is lower than a simple bound, no agent contributes in equilibrium. If instead $r$ exceeds this bound, there exists an equilibrium where all agents contribute. The equilibrium strategy profile that leads to full contribution prescribes contribution unless defection is observed.

To see why an agent contributes when she observes no defectors, note that if she contributes, then all her successors will also do so. If she instead defects, all her successors will also defect. Whether it makes sense to pay a cost (the contribution itself) to convince all her successors to contribute depends on the number of successors. However, agents are uncertain about their positions. Since only samples where all agents contribute are observed on the equilibrium path, an agent who observes such a sample is equally likely to be in any position. So she believes to be on average roughly in the middle of the se- 
quence. Then, whenever inducing contributions from half of the agents is more valuable than the cost of contributing, agents optimally choose to contribute. This corresponds to a return from contributions $r$ larger than approximately two.

Incentives off the equilibrium path depend on the sample size. When the sample size is larger than one, an agent who receives a sample that contains defection cannot herself prevent further defection by contributing (Lemma 1). Since she cannot affect her successors' decisions, she is better off responding to defection with defection. When instead the sample size is one, an agent who observes defection can restore contribution by contributing herself. This makes contributing after observing defection more appealing. We show that a (possibly) mixed equilibrium generates full contribution in this case too (Lemma 2). Agents contribute after observing contribution but randomize after observing defection (i.e. they forgive defection with positive probability).

We finally show that when the multiplication factor $r$ is too low to sustain full contribution, then no contribution can emerge (Lemma 3). This completes the proof of Proposition 1 . To see this, note that the profile of play that leads to full contribution provides strong incentives to contribute. An agent who contributes makes everybody after her contribute, while if she defects nobody does so. For any other profile of play, incentives are weaker. Then, if the return from contributions $r$ is too low to sustain full contribution, it is also too low to sustain any positive level of contribution in equilibrium.

Finally, we relax the assumption of complete position uncertainty by letting agents receive noisy signals about the position they are in. Lemma 4 shows that full contribution can still emerge, but it requires a higher multiplication factor $r$. We also discuss how our result applies to other social dilemmas. We focus on the most prominent example: the 
prisoners' dilemma. Lemma 5 shows that full cooperation is an equilibrium outcome.

Related Literature.- A large literature studies how cooperation can arise in social dilemmas. Most of the early work has focused on the prisoners' dilemma. Friedman's seminal work (1971) shows how sufficiently patient agents cooperate in an infinitely repeated prisoners' dilemma. ${ }^{1}$ Dal Bó (2005), Duffy and Ochs (2009) and several other papers provide experimental evidence in this direction. See Dal Bó and Fréchette (2018) for a recent survey on experimental studies about cooperation in infinitely repeated games.

Cooperation is not an equilibrium outcome of a finitely repeated prisoners' dilemma played by self-interested agents. ${ }^{2}$ However, experimental evidence shows that positive levels of cooperation also arise in finite settings (see Embrey et al. (2018) for a survey). Kreps et al. (1982) show that incomplete information about agents' types can explain cooperation. Long initial streaks of cooperation can occur when players believe that their opponents may be altruistic. However, defection eventually prevails. Andreoni (1990) and Fehr and Gächter (2000) suggest that cooperation can also arise when players' incentives are not exclusively determined by monetary payoffs. Andreoni focus on warm-glow effects from behaving altruistically, whereas Fehr and Gächter highlight the role of punishment. Our result differs in that we show cooperation can emerge when 1) there is a finite number of agents, 2) who are self-interested, and 3) each agent plays only once. ${ }^{3}$

\footnotetext{
${ }^{1}$ See Lockwood and Thomas (2002) and Guéron (2015) for a discussion on cooperation with gradualism and irreversibility in one's own level of cooperation.

${ }^{2}$ If instead the stage game has multiple Nash equilibria, cooperation can occur in equilibrium in finitely repeated games (see Benoit and Krishna (1985) and Benoit and Krishna (1987)).

${ }^{3} \mathrm{~A}$ different line of work studies repeated games that end with positive probability after each round (see Samuelson (1987) and Neyman (1999) for theoretical models, and Normann and Wallace (2012) for experimental evidence). Our model differs from this line of research in two main aspects: in our setting each agent moves only once and the total duration of the game is deterministic. Instead, a repeated game with a termination rule finishes in finite time almost surely, but lasts for any arbitrary large number of
} 
We study an environment with both position uncertainty and direct observation of predecessors' actions. Previous work on games with position uncertainty typically takes a different approach: there exists a principal who can choose flexibly which information about past actions to reveal to agents. We instead borrow from the literature on observational learning: agents directly observe the actions of others. Our environment with direct observation of actions is natural, but adds several hurdles, especially off the equilibrium. To see this, consider the related work by Nishihara (1997). He shows how a planner can induce cooperation in a prisoners' dilemma with position uncertainty. The planner must immediately inform all agents when defection occurs. On the equilibrium path, agents cooperate for the same reason as they contribute in our paper: to induce potential successors to also do so. Off the equilibrium path, all agents in Nishihara (1997) are immediately informed when defection occurs. Then, no agent can convince his successors to cooperate.

We focus instead on environments where observability is local and unconditional: agents observe their immediate predecessors' actions. Then, an agent who observes defection may prevent further defection by choosing to contribute. Our approach is different on this dimension also relative to Gershkov and Szentes (2009), Doval and Ely (2016) and Salcedo (2017). Gershkov and Szentes (2009) focus on optimal voting schemes and study the protocol a principal should choose to induce voters to acquire costly information and reveal it truthfully. In more general games, Doval and Ely (2016) and Salcedo (2017) study which recommendations a principal should convey for players to select the socially optimal action. In all these papers, as in Nishihara (1997), there is a principal who observes past actions and decides whether and how to reveal this information. The natural enviperiods with positive probability. 
ronment of direct observability makes the off the equilibrium analysis harder. We show how to solve for this difficulty, and characterise the maximum level of contributions for any return from contributions $r$.

Previous work on the voluntary provision of public goods studies how the timing of contributors' moves can determine the total amount that a principal can raise. In Varian's (1994) model, sequential timing lowers total contributions. However, experimental evidence (Andreoni et al. (2002) and Gächter et al. (2010)) suggests that sequential mechanisms may raise more funds than simultaneous ones. Andreoni (1998), Romano and Yildirim (2001), Vesterlund (2003) and Potters et al. (2005) argue that the sequential structure allows for the strategic release of information over time.

\section{The Model}

Let $\mathcal{I}=\{1, \ldots, n\}$ be a set of risk-neutral agents. Agents are exogenously placed in a sequence that determines the order of play. The random variable $Q$ assigns each agent a position in the sequence. Let $q:\{1,2, \ldots, n\} \rightarrow\{1,2, \ldots, n\}$ be a permutation and $\mathcal{Q}$ be the set of all possible permutations. We assume that all permutations are ex-ante equally likely: $\operatorname{Pr}(Q=q)=1 / n$ ! for all $q \in \mathcal{Q}{ }^{4}$ Agent $i^{\prime}$ s position is thus denoted by $Q(i)$.

When it is her turn to play, agent $i \in \mathcal{I}$ observes a sample of her predecessors' actions. She then chooses one of two actions $a_{i} \in\{C, D\}$. Action $a_{i}=C$ means contributing a fixed amount 1 to a common pool, while defection $\left(a_{i}=D\right)$ means investing $0 .^{5}$ Af-

\footnotetext{
${ }^{4}$ This setup corresponds to the case of symmetric position beliefs as defined in Monzón and Rapp (2014).

${ }^{5}$ The focus on a binary action space is made for simplicity. All our results extend to a setting in which agents can choose their level of contribution $C_{i}$ from a finite grid $\left\{0, \ldots, C_{\max }\right\}$. In particular, the social optimum in which all agents contribute the amount $C_{\max }$ is an equilibrium of the game.
} 
ter all players choose an action, the total amount invested gets multiplied by the return from contributions parameter $r$, and then equally shared among all agents. Let $G_{-i}=$ $\sum_{j \neq i} \mathbb{1}\left\{a_{j}=C\right\}$ denote the number of opponents who contribute, so $G_{-i} \in\{0, \ldots, n-1\}$. Payoffs $u\left(a_{i}, G_{-i}\right)$ can thus be expressed as:

$$
u_{i}\left(C, G_{-i}\right)=\frac{r}{n}\left(G_{-i}+1\right)-1 \quad \text { and } \quad u_{i}\left(D, G_{-i}\right)=\frac{r}{n} G_{-i} .
$$

We assume that $1<r<n$ so although contribution by all agents is socially optimal, each agent strictly prefers to defect for any given $G_{-i}$. Thus, payoffs from the public good are standard (see for instance Varian (1994), Potters et al. (2005), or Gächter et al. (2010)).

\subsection{Sampling}

Let $h_{t}=\left(a_{1}, a_{2}, \ldots, a_{t-1}\right)$ denote a possible history of actions up to period $t-1$. The random variable $H_{t}$ with realizations $h_{t} \in \mathcal{H}_{t}=\{C, D\}^{t-1}$ indexes all possible nodes in position $t$. Let $\mathcal{H}_{1}=\{\varnothing\}$.

Before choosing an action, each agent observes how many of her $m \geq 1$ immediate predecessors contributed. Agents in positions 1 to $m$ have less than $m$ predecessors, so they observe less than $m$ actions. A sample $\xi=\left(\xi^{\prime}, \xi^{\prime \prime}\right)$ is a pair, where the first component states the number of agents sampled, and the second component is the number of 
contributors in the sample. ${ }^{6}$ Formally, $\xi_{t}: \mathcal{H}_{t} \rightarrow \Xi=\mathbb{N}^{2}$ is given by

$$
\xi_{t}\left(h_{t}\right)=\left(\min \{m, t-1\}, \sum_{\tau=\max \{1, t-m\}}^{t-1} \mathbb{1}\left\{a_{\tau}=C\right\}\right) .
$$

The first agent in the sequence observes nobody's action, so she receives sample $\xi_{1}=$ $(0,0)$. Agents in positions 2 to $m$ observe the actions of all their predecessors. Thus, the first $m$ agents can infer their exact position from the size of the sample that they receive.

\subsection{Equilibrium Concept and Beliefs}

Players face an extensive-form game with imperfect information. They observe a sample of past actions and do not know their position in the sequence. Thus, when asked to play, they must form beliefs both about their own position and about the play of their predecessors.

We use the notion of sequential equilibrium as in Kreps and Wilson (1982). Agent $i$ 's strategy is a function $\sigma_{i}(C \mid \xi): \Xi \rightarrow[0,1]$ that specifies a probability of contributing given the sample received. Let $\sigma=\left\{\sigma_{i}\right\}_{i \in \mathcal{I}}$ denote a strategy profile and $\mu=\left\{\mu_{i}\right\}_{i \in \mathcal{I}}$ a system of beliefs. A pair $(\sigma, \mu)$ represents an assessment. Assessment $\left(\sigma^{*}, \mu^{*}\right)$ is a sequential equilibrium if $\sigma^{*}$ is sequentially rational given $\mu^{*}$, and $\mu^{*}$ is consistent given $\sigma^{*}$.

Agents form beliefs about their position in the game, and also about the history of actions that led to this position. Let $\mathcal{H}=\cup_{t=1}^{n} \mathcal{H}_{t}$ be the list of all possible histories. Given a profile of play $\sigma$, let $\mu_{i}$ denote agent $i$ 's beliefs about the history of play: $\mu_{i}(h \mid \xi)$ :

\footnotetext{
${ }^{6}$ For example, let $h_{5}=(C, C, D, C)$ be the history of actions up to period 4 and let $m=2$. Agents in positions 1 to 5 receive the following samples before they play: $\xi_{1}=(0,0), \xi_{2}=(1,1), \xi_{3}=(2,2)$, $\xi_{4}=(2,1)$, and $\xi_{5}=(2,1)$. Note that samples are unordered.
} 
$\mathcal{H} \times \Xi \rightarrow[0,1]$ with $\sum_{h \in \mathcal{H}} \mu_{i}(h \mid \xi)=1$ for all $\xi \in \Xi$.

To better illustrate how beliefs are formed, consider a game with only three agents and a sample of size one. When asked to play, an agent knows that there are seven possible histories of past play: $\{\varnothing,(C),(D),(C, C),(C, D),(D, C),(D, D)\}$. Each point in Figure 1 corresponds to one such history. ${ }^{7}$ After receiving the sample, agents form beliefs about the history of past play. An agent who observes $\xi=(0,0)$ realises that the history of past play is $h_{1}=\varnothing$ and thus she is in the first position (the square in Figure 1). Instead, an agent who observes $\xi=(1,1)$ knows that she is not in the first position. She may be in any position with a history of play that features contribution as last action (the circles in Figure 1). Similarly, an agent who observes $\xi=(1,0)$ knows that the history of play features defection as last action (the three triangles).

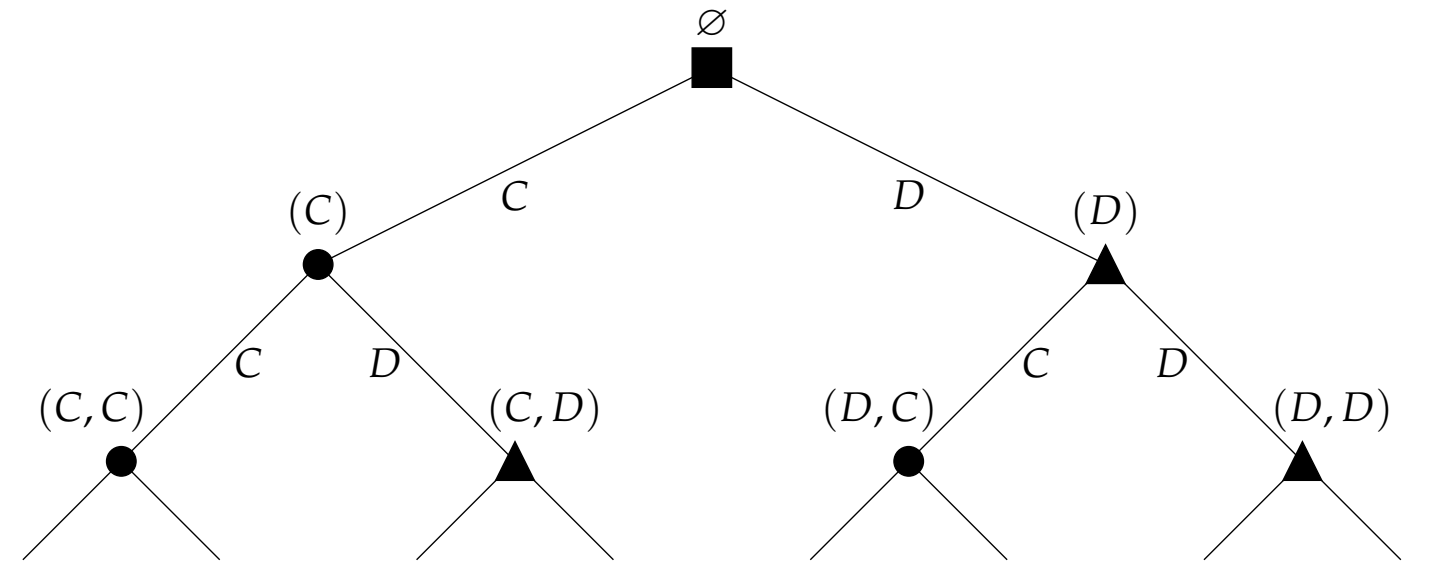

Figure 1: All Possible Histories of Past Play with 3 Agents

\footnotetext{
${ }^{7}$ Figure 1 represents all possible histories of past play, but does not represent the game tree. The game tree is significantly larger. It features an initial move by Nature who chooses the ordering, followed by a sequence of three moves for each possible order.
} 


\section{Full Contribution in Equilibrium}

In this section we present our main result. When the return from contributions is high enough, there exists an equilibrium with full contribution. If instead the return is below the threshold, then nobody contributes.

Proposition 1. Full Contribution with Position UnCERTAinty.

(a) If $r \geq 2\left(1+\frac{m-1}{n-m+1}\right)$, then there exists an equilibrium in which all agents contribute.

(b) If instead $r<2\left(1+\frac{m-1}{n-m+1}\right)$, then no agent contributes in equilibrium.

We present the proof of Proposition 1 through a series of lemmas. Lemma 1 shows that full contribution is an equilibrium outcome when agents observe the actions of more than one predecessor $(m \geq 2)$. Next, Lemma 2 proves that this is also true when agents only observe the action of their immediate predecessor $(m=1)$. Finally, Lemma 3 shows that when the return from contributions is too low, then nobody contributes.

\subsection{Samples of Size $\mathbf{m} \geq \mathbf{2}$}

The equilibrium with full contribution features a simple profile of play: agents contribute unless they observe a defection. Let $\Xi^{C}$ include all samples without defection. This set consists of all samples where the number of observed individuals $\xi^{\prime}$ is equal to the number of observed individuals who contributed $\xi^{\prime \prime}$. That is, $\xi=\left(\xi^{\prime}, \xi^{\prime \prime}\right) \in \Xi^{C} \Leftrightarrow \xi^{\prime}=\xi^{\prime \prime}$. Note that the first agent in the sequence receives a sample without defection: $(0,0) \in \Xi^{C}$.

In what follows we let $\left\{\sigma^{k}\right\}_{k=1}^{\infty}$ be a sequence of perturbed strategy profiles that places equal probability on all deviations. This sequence induces beliefs $\mu_{i}^{k}$ with $\lim _{k \rightarrow \infty} \mu^{k}=$ 
$\mu^{*}{ }^{8}$

LEMma 1. Full CONTRIBUtion With SAMPLE SizE $\mathbf{m} \geq \mathbf{2}$. Consider the following profile of play:

$$
\sigma_{i}^{*}(C \mid \xi)=\left\{\begin{array}{ll}
1 & \text { if } \xi \in \Xi^{C} \\
0 & \text { if } \xi \notin \Xi^{C}
\end{array} \quad \text { for all } i \in \mathcal{I}\right.
$$

Then $\left(\sigma^{*}, \mu^{*}\right)$ is a sequential equilibrium of the game whenever $r \geq 2[1+(m-1) /(n-m+1)]$.

The next two sections provide the intuition for this result for samples without and with defection, respectively (see Appendix A.1 for the proof).

\subsubsection{Samples without defection}

Consider first an agent who observes a sample without defection: $\xi \in \Xi^{C}$. This occurs on the equilibrium path, so the agent infers that all her predecessors contributed. She knows that if she contributes, then all subsequent players will also do so. Therefore, $i$ 's expected payoff from contributing is $E_{\mu^{*}}\left[u\left(C, G_{-i}\right) \mid \xi\right]=r-1$ for all $\xi \in \Xi^{C}$. This payoff is independent of agent $i$ 's beliefs about her position in the sequence.

An agent's payoff from defecting does depend on her beliefs about her position. To see this, assume first that agent $i$ knows that she is in position $Q(i)=t$. All her predecessors contributed, but none of her successors will do so (since she herself does not contribute). Then, exactly $t-1$ players contribute. The payoff from defecting is simply $E_{\tilde{\mu}}\left[u\left(D, G_{-i}\right) \mid \xi, Q(i)=t\right]=(r / n)(t-1)$ for all $\xi \in \Xi^{C}$, where $\tilde{\mu}$ are the beliefs induced by the deviation. Figure 2 illustrates agent $i$ 's payoffs as a function of her posi-

\footnotetext{
${ }^{8}$ Specifically, $\sigma_{i}^{k}(C \mid \xi)=1-k^{-1}$ for all $\xi \in \Xi^{C}$ and $\sigma_{i}^{k}(C \mid \xi)=k^{-1}$ for all $\xi \notin \Xi^{C}$. We consider equivalent sequences of perturbed strategies for all results in this paper, so we omit them hereafter.
} 
tion. For agents placed early in the sequence, the payoff from contributing is larger than from defecting. Later agents prefer defection though. Then if agents knew their position, contribution would unravel.

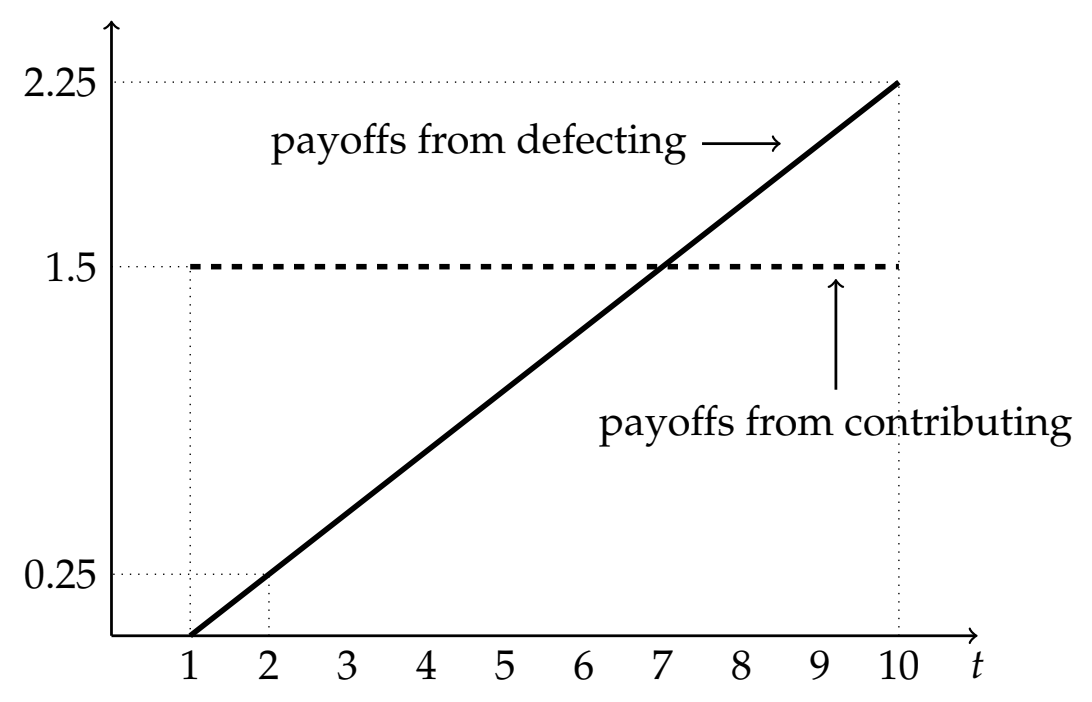

Figure 2: Payoffs Conditional on Position and on Sample $\xi \in \Xi^{C}(r=2.5, n=10)$

The fact that players do not know their position can make every agent who observes only contributors willing to contribute. Consider an agent who observes $m$ agents contributing. Then, she knows that she is not in the first $m$ positions. Other than that, she has an equal probability of being in any position $\{m+1, \ldots, n\}$. Thus, her expected position is $(n+m+1) / 2$, i.e., she expects $(n+m-1) / 2$ agents to have already contributed. Therefore, the expected payoff from defecting is $E_{\tilde{\mu}}\left[u\left(D, G_{-i}\right) \mid \xi=(m, m)\right]=$ $(r / n)(n+m-1) / 2$. Contribution then requires $r-1 \geq(r / n)(n+m-1) / 2$, which simplifies to the following condition:

$$
r \geq 2\left(1+\frac{m-1}{n-m+1}\right)
$$


If instead the sample contains $\xi^{\prime}<m$ total actions, the agent knows that she is in position $\xi^{\prime}+1$. The number of agents who contributed so far is $\xi^{\prime}$. Therefore, the expected payoff from defecting is even lower: $(r / n) \xi^{\prime}<(r / n)(n+m-1) / 2$. Equation (1) thus also guarantees that agents in the first $m$ positions contribute.

\subsubsection{Samples with defection}

Consider next an agent who observes a sample with defection: $\xi \notin \Xi^{C}$. The equilibrium profile of play requires that she herself defects. The key to understanding why this is optimal is that an agent who observes defection cannot prevent her successors from defecting. We explain this claim case by case. First, take an agent in one of the first $m$ positions who receives a sample with at least one defector. Her immediate successor will also receive a sample with (at least) one defector, and will thus defect. Next, consider an agent who receives a sample with more than one defector. Her successor will also defect. For example, let $m=2$ and assume that agent $i$ receives sample $\xi=(2,0)$. The only histories $h \in \mathcal{H}$ consistent with this sample are those with $h_{t}=(\ldots, D, D)$. Then, the next period's history will be of the form $h_{t+1}=\left(\ldots, D, a_{i}\right)$. It follows that, regardless of agent $i$ 's choice, her successor will defect, and so will all the remaining players.

So an agent may hope to prevent further defection only after receiving a sample $\xi=(m, m-1)$; that is, observing $m$ actions with only one defection. For this to be true, the sole defector in the sample must be in position $t-m$. Otherwise, her successor would still observe one defection in his sample, and this would make him defect. However, an agent who observes a sample $\xi=(m, m-1)$ assigns zero probability to the player defecting being in position $t-m$. To see this, consider again the case with $m=2$. When 
the agent receives the sample $\xi=(2,1)$, she does not know in principle whether it was her immediate predecessor $(t-1)$, or the one before $(t-2)$ who defected. If it was her immediate predecessor, the history of play is of the form $h_{t}=(\ldots, C, D)$. This history is consistent with only one mistake with respect to the equilibrium strategy. If it was the agent before, then $h_{t}=(\ldots, D, C)$. At least two mistakes occurred: not only that someone defected (which does not happen on the equilibrium path), but also that somebody contributed after observing defection. Therefore, the agent assigns zero probability to history being of the form $h_{t}=(\ldots, D, C)$.

It follows that an agent who observes defection cannot affect her successors' actions, regardless of the value of $r$. This explains why agents always defect after observing a defection in their sample.

\subsection{Samples of Size $\mathbf{m}=\mathbf{1}$}

The case when only one agent is observed requires a separate discussion. When $m=1$, an agent who observes defection can prevent further defection by choosing to contribute. Then, if the return from contributions is too high, the simple strategy of contributing unless defection is observed cannot be an equilibrium. Agents would find it optimal to contribute after observing contribution, but would not find it optimal to defect after observing a defection. When $r$ is too high, an agent who observes defection finds the cost of contributing worth paying in order to induce all her successors to contribute. Then, she would choose to contribute instead of defecting. However, since $r<n$, the strategy profile in which all agents contribute is not an equilibrium, because players who observe 
contribution would deviate to defecting. This implies that a pure strategy equilibrium with full contribution cannot arise.

When the multiplication factor $r$ is large, full contribution can arise in a mixed strategy equilibrium. We construct a profile of play in which agents respond to contribution with contribution and "forgive" defection with probability $\gamma \in[0,1)$. The possibility of future forgiveness makes defecting more attractive: successors may restore contribution. This makes the threat of defection credible off the equilibrium path.

Lemma 2. Full Contribution With SAMPle Size $\mathbf{m}=\mathbf{1}$. Consider the following profile of play:

$$
\sigma_{i}^{*}(C \mid \xi)=\left\{\begin{array}{ll}
1 & \text { if } \xi \in\{(0,0),(1,1)\} \\
\gamma \quad \text { if } \xi=(1,0)
\end{array} \quad \text { for all } i \in \mathcal{I}\right.
$$

For any $r \geq 2$ there exists $\gamma \in[0,1)$ such that $\left(\sigma^{*}, \mu^{*}\right)$ is a sequential equilibrium of the game.

See Appendix A.2 for the proof.

In line with Lemma 1, there is an equilibrium with full contribution whenever $r \geq 2$. For $r \in[2,3-3 /(n+1)]$ the equilibrium is pure, while for $r \in(3-3 /(n+1), n)$ the equilibrium is mixed $(\gamma>0)$.

\subsection{Comparative Statics}

Together, the conditions that sustain full contribution when $m \geq 2$ and when $m=1$ prove the first part of Proposition 1: an equilibrium with full contribution exists for any $m \geq 1$ and any $r \geq 2[1+(m-1) /(n-m+1)]$. Corollary 1 immediately follows.

COROLLARY 1. The lower bound on $r$ for full contribution strictly increases in $m$. 
Thus, the range on the multiplication factor $r$ consistent with full contribution is the largest for $m=1 .^{9}$

\subsection{No Contribution for Low $r$}

We complete the proof of Proposition 1 by showing that when the return from contributions is not high enough to achieve full contribution, then nobody contributes.

LEMma 3. No CONTRIBUtion FOR LOW $r$. Whenever $r<2[1+(m-1) /(n-m+1)]$, no agent contributes in equilibrium.

See Appendix A.3 for the proof.

The intuition is as follows. Assume that contribution occurs in equilibrium and consider the incentives of an agent $i$ who contributes after observing a sample $\xi$. Her benefit from contributing is given by contributions from successors who would otherwise defect. The cost is 1 , the contribution itself.

The equilibrium profile we propose in Lemma 1 provides strong incentives to contribute on the equilibrium path. An agent who contributes gets everybody after her to do so. On the contrary, if she defects, nobody after her contributes. Then, for any other possible profile of play, the number of successors who contribute instead of defecting because of agent $i$ 's contribution is at most everybody after $i$. A necessary condition for $i$ to

\footnotetext{
${ }^{9}$ If $m=0$ (i.e., agents get no information about their predecessors' actions) the setting becomes analogous to a simultaneous game and thus an equilibrium with full contribution does not exist.
} 
contribute after observing sample $\xi$ is thus that:

$$
\overbrace{\frac{r}{n} E_{\mu}\left[\sum_{\tau \geq Q(i)} \mathbb{1}\left\{a_{\tau}=C\right\} \mid a_{i}=C, \xi\right]}^{\text {upper bound on benefit from contributing }} \geq \overbrace{1}^{\substack{\text { cost of } \\ \text { contributing }}} .
$$

Therefore, an agent who contributes expects that there are at least $n / r-1$ contributors among her successors: $E_{\mu}\left[\sum_{\tau>Q(i)} \mathbb{1}\left\{a_{\tau}=C\right\} \mid a_{i}=C, \xi\right] \geq n / r-1$.

The average number of successors who contribute follows a simple rule (see Appendix A.3 for further details). When $m=1$, it cannot exceed $n / 2-1$. Then, contribution cannot emerge if $r<2$. When $m>1$, the condition becomes $r<2[1+(m-1) /(n-m+$ $1)]$.

\section{Extensions}

The following extensions shed further light on how cooperation can emerge with position uncertainty. First, we show that our result is robust to agents having noisy information about their position. Second, we discuss how our framework sustains full cooperation in a prisoners' dilemma.

\subsection{Noisy Information on Positions}

Assume that before observing a sample of past actions, each agent receives a noisy signal about her position in the sequence. Let agent $i$ be in position $Q(i)=t$. Signal $S_{t}$, which 
takes values $s \in \mathcal{S}=\{1,2, \ldots, n\}$, follows:

$$
S_{t}= \begin{cases}t \quad \text { with probability } \lambda \in(1 / n, 1) \\ \tau \quad \text { with probability }(1-\lambda) /(n-1) \quad \text { for all } \tau \in\{1, \ldots, n\} \text { with } \tau \neq t\end{cases}
$$

Therefore, an agent who receives signal $s$ and has not yet observed the sample of past actions believes that she is position $s$ with probability $\lambda$. She then observes the sample, updates her beliefs, and chooses to contribute or defect. A strategy is a map $\sigma_{i}(C \mid s, \xi)$ : $\mathcal{S} \times \Xi \rightarrow[0,1]$.

Lemma 4. NoIsy INFORMATION ON POSITIONS. Let $m \geq 2$. Consider the following profile of play:

$$
\sigma_{i}^{*}(C \mid s, \xi)=\left\{\begin{array}{ll}
1 & \text { if } \xi \in \Xi^{C} \\
0 & \text { if } \xi \notin \Xi^{C}
\end{array} \quad \text { for all } i \in \mathcal{I}\right.
$$

Then, $\left(\sigma^{*}, \mu^{*}\right)$ is a sequential equilibrium of the game if and only if

$$
r \geq 2 n\left[2+\frac{(n-1-m)(n-m)}{(n-1) /(1-\lambda)-m}\right]^{-1}
$$

See Appendix A.4 for the proof.

Intuitively, signals make agents less uncertain about their position, and thus in some cases less inclined to contribute. Condition (2) guarantees that an agent contributes even after receiving a signal $s=n$. She does so because with positive probability she is placed elsewhere in the sequence. Condition (2) defines a lower bound on $r$ that increases in $\lambda$; the stronger the signal about the player being in the last position, the higher the multipli- 
cation factor $r$ needed to convince her to contribute.

The following straightforward corollary shows that as long as signals are not perfectly informative, there is always a high enough multiplication factor $r$ that sustains full contribution as an equilibrium.

COROLlaRy 2. For all $\lambda<1$ there exists $r<n$ such that $\left(\sigma^{*}, \mu^{*}\right)$ is a sequential equilibrium of the game.

\subsection{Prisoners' Dilemma}

Consider the following simple modification to the model presented in Section 1. Agents sequentially choose whether to cooperate or defect. After all players have chosen their actions, each agent is matched to all of her $n-1$ opponents in a series of pairwise prisoners' dilemma interactions. Payoffs to agent $i$ from each interaction are shown in Table 1.

\begin{tabular}{c|c|cc} 
& \multicolumn{3}{c}{ opponent's action } \\
& & $C$ & $D$ \\
\cline { 2 - 4 } agent $i$ 's action & $C$ & 1 & $-l$ \\
& $D$ & $1+g$ & 0
\end{tabular}

Table 1: Payoffs to Agent $i$ in the Prisoners' Dilemma

As usual, $g>0$ represents the gain from defecting when the opponent cooperates, while $l>0$ represents the loss from cooperating when the opponent defects. Agent $i$ 's total payoffs are the sum of the payoffs from each pairwise interaction: $u_{i}\left(C, G_{-i}\right)=$ $G_{-i}-\left(n-1-G_{-i}\right) l$ and $u_{i}\left(D, G_{-i}\right)=(1+g) G_{-i}$.

Lemma 5 illustrates how full cooperation can emerge when agents play sequentially, are uncertain about their positions, and observe the actions of some of their immediate predecessors. 
Lemma 5. Full Cooperation in a Prisoners' Dilemma. Let $m \geq 2$. Consider the following profile of play:

$$
\sigma_{i}^{*}(C \mid \xi)=\left\{\begin{array}{ll}
1 & \text { if } \xi \in \Xi^{C} \\
0 & \text { if } \xi \notin \Xi^{C}
\end{array} \quad \text { for all } i \in \mathcal{I}\right.
$$

Then $\left(\sigma^{*}, \mu^{*}\right)$ is a sequential equilibrium of the game whenever $g \leq 1-2 m /(n+m-1)$.

The proof closely follows that of Lemma 1 (see Appendix A.5 for the details).

\section{Discussion}

We describe a natural environment that fosters cooperation in social dilemmas. In social dilemmas, which are at the core of many economic problems, it is socially optimal that agents cooperate, but they have private incentives not to do so. A large body of work has studied several ways to sustain cooperation. We show how full cooperation can emerge in a context where it may seem ex-ante hard to achieve: one-shot games with a finite number of self-interested players.

We present our results in a public good game. Players choose sequentially whether to contribute or defect, are uncertain about their position in the sequence, and observe a sample of their predecessors' choices. Because of partial observability of past actions, agents contribute to induce others to also do so. Full contribution can emerge when the return from contributions is above a threshold approximately equal to two. Multiplication factors both below and above two have been commonly used in experimental 
work (Zelmer (2003) reports that multiplication factors typically range from 1.6 to 4 ). In fundraising activities with multiplication factors lower than the threshold, charities can consider partially matching agents' contributions to exploit our result. We also show how our result applies to a prisoners' dilemma.

Although outside of the scope of this paper, future experimental work can investigate whether, as predicted by our model, position uncertainty and partial observation of others' actions foster cooperation.

Andrea Gallice

University of Turin (ESOMAS Department) and Collegio Carlo Alberto

Ignacio Monzón

Collegio Carlo Alberto

\section{References}

Andreoni, J. (1990). 'Impure altruism and donations to public goods: A theory of warmglow giving?', Economic Journal, vol. 100(401), pp. 464-77.

Andreoni, J. (1998). 'Toward a theory of charitable fund-raising', Journal of Political Economy, vol. 106(6), pp. 1186-1213.

Andreoni, J., Brown, P.M. and Vesterlund, L. (2002). ‘What makes an allocation fair? some experimental evidence', Games and Economic Behavior, vol. 40(1), pp. 1-24. 
Benoit, J.P. and Krishna, V. (1985). 'Finitely repeated games', Econometrica, vol. 53(4), pp. $905-22$.

Benoit, J.P. and Krishna, V. (1987). 'Nash equilibria of finitely repeated games', International Journal of Game Theory, vol. 16(3), pp. 197-204.

Dal Bó, P. (2005). 'Cooperation under the shadow of the future: Experimental evidence from infinitely repeated games', American Economic Review, vol. 95(5), pp. 1591-1604.

Dal Bó, P. and Fréchette, G.R. (2018). 'On the determinants of cooperation in infinitely repeated games: A survey', Journal of Economic Literature, vol. 56(1), pp. 60-114.

Doval, L. and Ely, J. (2016). 'Sequential information design', Working Paper.

Duffy, J. and Ochs, J. (2009). 'Cooperative behavior and the frequency of social interaction', Games and Economic Behavior, vol. 66(2), pp. 785-812.

Embrey, M., Fréchette, G.R. and Yuksel, S. (2018). ‘Cooperation in the finitely repeated prisoners dilemma', The Quarterly Journal of Economics, vol. 133(1), pp. 509-551.

Fehr, E. and Gächter, S. (2000). 'Cooperation and punishment in public goods experiments', The American Economic Review, vol. 90(4), pp. 980-994.

Friedman, J.W. (1971). 'A non-cooperative equilibrium for supergames', The Review of Economic Studies, vol. 38(1), pp. 1-12.

Gächter, S., Nosenzo, D., Renner, E. and Sefton, M. (2010). 'Sequential vs. simultaneous contributions to public goods: Experimental evidence', Journal of Public Economics, vol. 94(78), pp. 515-522. 
Gershkov, A. and Szentes, B. (2009). 'Optimal voting schemes with costly information acquisition', Journal of Economic Theory, vol. 144(1), pp. 36 - 68.

Guéron, Y. (2015). 'Failure of gradualism under imperfect monitoring', Journal of Economic Theory, vol. 157(C), pp. 128-145.

Kreps, D.M., Milgrom, P., Roberts, J. and Wilson, R. (1982). 'Rational cooperation in the finitely repeated prisoners' dilemma', Journal of Economic Theory, vol. 27(2), pp. 245-252.

Kreps, D.M. and Wilson, R. (1982). 'Sequential equilibria', Econometrica, vol. 50(4), pp. 863-894.

Lockwood, B. and Thomas, J.P. (2002). 'Gradualism and irreversibility', The Review of Economic Studies, vol. 69(2), pp. 339-356.

Monzón, I. and Rapp, M. (2014). ‘Observational Learning with Position Uncertainty', Journal of Economic Theory, vol. 154, pp. 375-402.

Neyman, A. (1999). 'Cooperation in repeated games when the number of stages is not commonly known', Econometrica, vol. 67(1), pp. 45-64.

Nishihara, K. (1997). 'A resolution of n-person prisoners' dilemma', Economic Theory, vol. 10(3), pp. 531-540.

Normann, H.T. and Wallace, B. (2012). 'The impact of the termination rule on cooperation in a prisoners dilemma experiment', International Journal of Game Theory, vol. 41(3), pp. $707-718$. 
Potters, J., Sefton, M. and Vesterlund, L. (2005). 'After you-endogenous sequencing in voluntary contribution games', Journal of Public Economics, vol. 89(8), pp. 1399-1419.

Romano, R. and Yildirim, H. (2001). 'Why charities announce donations: a positive perspective', Journal of Public Economics, vol. 81(3), pp. 423-447.

Salcedo, B. (2017). 'Interdependent choices', Working Paper.

Samuelson, L. (1987). 'A note on uncertainty and cooperation in a finitely repeated prisoner's dilemma', International Journal of Game Theory, vol. 16(3), pp. 187-195.

Varian, H.R. (1994). 'Sequential contributions to public goods', Journal of Public Economics, vol. 53(2), pp. 165-186.

Vesterlund, L. (2003). 'The informational value of sequential fundraising', Journal of Public Economics, vol. 87(3-4), pp. 627-657.

Zelmer, J. (2003). 'Linear public goods experiments: A meta-analysis', Experimental Economics, vol. 6(3), pp. 299-310.

\section{A. Proofs}

\section{A.1 Proof of Lemma 1}

Agents play only once. They do not observe the entire history of past play and are uncertain about their position in the sequence. Before choosing whether to contribute or defect, 
they observe a sample of past play $\xi$. Samples $\xi \in \Xi$ index information sets, which typically contain several nodes. We next verify that the strategy profile of play defined in Lemma 1 is indeed an equilibrium. To do so, we check that no agent has an information set where a profitable deviation exists. ${ }^{10}$ In this sense, our approach is analogous to the one-shot deviation principle. In our model, however, as each agent plays only once, possible deviations in one information set have no effect on payoffs that stem from a different information set.

Consider first a sample $\widetilde{\zeta} \in \Xi^{C}$. Agent $i^{\prime}$ s alternative strategy $\tilde{\sigma}_{i}$ is equal to $\sigma_{i}^{*}$ except that it specifies defection after observing sample $\widetilde{\xi}$, i.e., $\tilde{\sigma}_{i}(C \mid \widetilde{\xi})=0$. Induced beliefs are denoted by $\tilde{\mu}$. Agent $i$ contributes as long as:

$$
\begin{aligned}
E_{\mu^{*}}\left[u_{i}\left(a_{i}, G_{-i}\right) \mid \widetilde{\zeta}\right] & \geq E_{\tilde{\mu}}\left[u_{i}\left(a_{i}, G_{-i}\right) \mid \widetilde{\zeta}\right] \\
r-1 & \geq \frac{r}{n} E_{\tilde{\mu}}[Q(i)-1 \mid \widetilde{\zeta}]
\end{aligned}
$$

where the last step follows directly from our discussion in Section 2.1.1.

If the sample $\widetilde{\zeta}$ includes $m$ actions, agent $i$ understands that she is equally likely to be in any position but the first $m$. Her expected position is

$$
E_{\tilde{\mu}}[Q(i) \mid \widetilde{\xi}]=\frac{1}{n-m} \sum_{t=m+1}^{n} t=\frac{n+m+1}{2} .
$$

\footnotetext{
${ }^{10}$ The remaining proofs in the paper follow the same logic.
} 
Equation (3) becomes:

$$
r-1 \geq \frac{r}{n}\left(\frac{n+m+1}{2}-1\right) \Leftrightarrow r \geq 2\left(1+\frac{m-1}{n-m+1}\right)
$$

As discussed in section 2.1.1, after receiving a sample without defection and with $\widetilde{\xi}^{\prime}<$ $m$ total actions, the agent learns her position. Payoffs from defecting are even lower, so condition (1) suffices.

Consider next samples with defection $\xi \notin \Xi^{C}$. Let $\mathcal{H}^{D}$ denote the set of all nodes that generate a sample with defection and where successors always defect. That is, $h_{t} \in \mathcal{H}^{D}$ whenever 1) $\xi_{t}\left(h_{t}\right) \notin \Xi^{C}$ and 2) $a_{\tau}=D$ for all $\tau>t$, regardless of $a_{t}$. Note that if an agent's successor chooses $a_{t+1}=D$ after the agent contributes $\left(a_{t}=C\right)$ then $h_{t} \in \mathcal{H}^{D}$.

The following intermediate lemma provides a simple characterization of nodes that 1 ) generate samples with defection and 2) allow the agent to affect her successors' actions. Lemma 6. Assume that $\xi_{t}\left(h_{t}\right) \notin \Xi^{C}$ and $h_{t} \notin \mathcal{H}^{D}$. Then $t>m$ and $\xi_{t}\left(h_{t}\right)=(m, m-1)$. Moreover, $a_{t-m}=D$, and $a_{\tau}=C$ for $t-m+1 \leq \tau \leq t-1$. So $h_{t}$ looks as follows:

$$
h_{t}=(\ldots, \overbrace{D, C, C, \ldots, C}^{\text {agents sampled }})
$$

Proof. First, assume $h_{t}$ is such that $t \leq m$. Then, regardless of the action of the agent in position $t, \xi_{t+1}\left(h_{t+1}\right) \notin \Xi^{C}$. Then the agent in position $t+1$ defects. Second, assume that $h_{t}$ is such that $t>m$ and that more than one agent defects in the sample: $\xi_{t}\left(h_{t}\right) \neq$ $(m, m-1)$. Then, regardless of $a_{t}$, the agent in position $t+1$ still observes at least one defection. So $a_{t+1}=D$. Third, assume that $\xi_{t}\left(h_{t}\right) \neq(m, m-1)$ and $a_{t-m}=C$. Then again 
there is a defection in $\xi_{t+1}$. So $a_{t+1}=D$.

With Lemma 6 in hand it is easy to show that an agent can never affect her successors' actions if she observes defection. An agent who receives a sample $\xi=(m, m-1)$ must form beliefs about the nodes she may be in. Any node $h_{t} \in \mathcal{H}^{D}$ has at least $m$ deviations. Consider instead the node $h_{t}=(C, \ldots, C, D)$, that is $a_{\tau}=C$ for all $\tau \leq t-2$ and $a_{t-1}=D$. This node has only one deviation. Then, $\sum_{h_{t} \in \mathcal{H}^{D}} \mu^{*}\left(h_{t} \mid \xi\right)=1$ for all $\xi \notin \Xi^{C}$. Then, an agent who observes defection never believes that she can prevent further defection.

\section{A.2 Proof of Lemma 2}

Let $\sigma^{*}$ be defined as in the statement of the lemma. Take a generic sample $\tilde{\xi}$ and build two profiles of play. First, $\sigma^{C}=\left(\sigma_{i}^{C}, \sigma_{-i}^{*}\right)$ with $\sigma_{i}^{C}(C \mid \xi=\tilde{\xi})=1$ and $\sigma_{i}^{C}=\sigma_{i}^{*}$ for all other samples. Second, $\sigma^{D}=\left(\sigma_{i}^{D}, \sigma_{-i}^{*}\right)$ with $\sigma_{i}^{D}(C \mid \xi=\tilde{\xi})=0$ and $\sigma_{i}^{D}=\sigma_{i}^{*}$ for all other samples. Induced beliefs are denoted by $\mu^{C}$ and $\mu^{D}$.

Let $v_{t}(\gamma)$ represent the (expected) number of additional contributors an agent gets by contributing rather than defecting after observing sample $\tilde{\xi}$ and conditional on being in position $t .{ }^{11}$ Similarly, let $f_{t}(\gamma)$ represent the likelihood of being in position $t$ after observing defection (sample $\xi=(1,0)$ ):

$$
\begin{aligned}
& v_{t}(\gamma) \equiv E_{\mu^{C}}\left[G_{-i} \mid \xi=\tilde{\xi}, Q(i)=t\right]-E_{\mu^{D}}\left[G_{-i} \mid \xi=\tilde{\xi}, Q(i)=t\right]+1 \\
& f_{t}(\gamma) \equiv \mu^{*}[Q(i)=t \mid \xi=(1,0)]
\end{aligned}
$$

\footnotetext{
${ }^{11}$ An agent who contributes generates a direct effect (her own contribution) and an indirect one, as she may affect the actions of successors. The last term in the definition of $v_{t}(\gamma)$ represents the agent's own contribution. That is why $v_{n}(\gamma)=1$ for any $\gamma \in[0,1]$.
} 
Then, the agent in the first position in the sequence - who receives sample $\xi=(0,0)$ - contributes whenever $(r / n) v_{1}(\gamma)-1 \geq 0$. Instead, an agent who receives a sample $\xi=(1,1)$ contributes whenever $(r / n)\left[\sum_{t=2}^{n}(n-1)^{-1} v_{t}(\gamma)\right]-1 \geq 0$. Finally, an agent who receives sample $\xi=(1,0)$ is indifferent when $(r / n)\left[\sum_{t=2}^{n} f_{t}(\gamma) v_{t}(\gamma)\right]-1=0$. The following intermediate lemma characterises these functions.

LEMMA 7. The functions $v_{t}(\gamma):[0,1] \rightarrow \mathbb{R}$ and $f_{t}(\gamma):[0,1] \rightarrow[0,1]$ are as follows:

$$
\begin{aligned}
& v_{t}(\gamma)= \begin{cases}\gamma^{-1}\left[1-(1-\gamma)^{n-t+1}\right] & \text { if } \gamma \in(0,1] \\
n-t+1 & \text { if } \gamma=0\end{cases} \\
& f_{t}(\gamma)= \begin{cases}\frac{1-(1-\gamma)^{t-1}}{n-1-\frac{1-\gamma}{\gamma}\left(1-(1-\gamma)^{n-1}\right)} & \text { if } \gamma \in(0,1] \\
2 \frac{t-1}{n(n-1)} & \text { if } \gamma=0\end{cases}
\end{aligned}
$$

Moreover, $v_{t}(\gamma)$ and $f_{t}(\gamma)$ are continuous in $\gamma \in[0,1]$ and such that $v_{t}(\gamma)>v_{t+1}(\gamma)$ and $f_{t}(\gamma)<f_{t+1}(\gamma)$ for all $\gamma \in[0,1)$. Finally, $\partial v_{t}(\gamma) / \partial \gamma<0$ for $t<n$ and $\gamma<1$.

See Appendix A.2.1 for the proof.

By Lemma $7,(r / n) v_{1}(\gamma)>(r / n)\left[\sum_{t=2}^{n}(n-1)^{-1} v_{t}(\gamma)\right]$. So if agents contribute after observing $\xi=(1,1)$, they also do so after observing $\xi=(0,0)$.

We show next that the CDF given by $f_{t}(\gamma)$ first order stochastically dominates the uniform distribution given by $1 /(n-1)$. Note that $f_{t}(\gamma)$ is strictly increasing in $t$ for all $\gamma \in[0,1)$. Then, there exists $\tilde{t}$ such that $f_{t}(\gamma) \leq 1 /(n-1)$ for all $t \leq \tilde{t}$, and $f_{t}(\gamma) \geq$ $1 /(n-1)$ for all $t>\tilde{t}$. Therefore, $\sum_{\tau=2}^{t} f_{\tau}(\gamma) \leq \sum_{\tau=2}^{t} 1 /(n-1)$ for all $t \leq \tilde{t}$. Similarly, 
$\sum_{\tau=t}^{n} f_{\tau}(\gamma) \geq \sum_{\tau=t}^{n} 1 /(n-1)$ for all $t>\tilde{t}$. Then,

$$
\sum_{\tau=t}^{n} f_{\tau}(\gamma) \geq \sum_{\tau=t}^{n} \frac{1}{n-1} \Leftrightarrow 1-\sum_{\tau=2}^{t-1} f_{\tau}(\gamma) \geq 1-\sum_{\tau=2}^{t-1} \frac{1}{n-1} \Leftrightarrow \sum_{\tau=2}^{t-1} f_{\tau}(\gamma) \leq \sum_{\tau=2}^{t-1} \frac{1}{n-1}
$$

It follows that $\sum_{\tau=2}^{t} f_{\tau}(\gamma) \leq \sum_{\tau=2}^{t} 1 /(n-1)$ for all $t \geq \tilde{t}$. This, and the fact that $v_{t}(\gamma)$ is decreasing in $\gamma$, imply that $\sum_{t=2}^{n}(n-1)^{-1} v_{t}(\gamma) \geq \sum_{t=2}^{n} f_{t}(\gamma) v_{t}(\gamma)$. The previous expression holds with strict inequality if $\gamma \in[0,1)$.

Define $H(\gamma) \equiv(r / n)\left[\sum_{t=2}^{n} f_{t}(\gamma) v_{t}(\gamma)\right]-1$. Note that whenever $H(\gamma)<0$, an agent defects after observing $\xi=(1,0)$. Since $H(0)=(r / 3)(n+1) / n-1$, it follows that whenever $2 \leq r \leq 3-3 /(n+1)$, a pure equilibrium exists (the lower bound ensures that contribution follows contribution, the argument replicates the one discussed in the proof of Lemma 1 in the context $m=1)$. If instead $r>3-3 /(n+1)$, then $H(0)>0$. For those values of $r$, if $\gamma=0$ contributing after a defection is preferred. So no pure equilibrium can sustain full contribution. If instead $\gamma=1$, then $H(1)=r / n-1<0$. Note that $H(\gamma)$ is continuous in $\gamma \in[0,1]$, since both $f_{t}(\gamma)$ and $v_{t}(\gamma)$ are continuous in $\gamma \in[0,1]$. Then, there exists $\gamma \in(0,1)$ such that $H(\gamma)=0$. Solving explicitly for $H(\gamma)=0$ leads to:

$$
\frac{2}{\gamma}-\frac{(n-1)\left(1-(1-\gamma)^{n}\right)}{\gamma n-1+(1-\gamma)^{n}}=\frac{n}{r}
$$




\section{A.2.1 Proof of Lemma 7}

The expected number of opponents who cooperate, given a particular position and sample $\xi=\tilde{\xi}$ is given by:

$$
\begin{aligned}
E_{\mu^{c}}\left[G_{-i} \mid \xi=\tilde{\xi}, Q(i)=t\right] & =E_{\mu^{c}}\left[\sum_{\tau=1}^{t-1} \mathbb{1}\left\{a_{\tau}=C\right\}+\sum_{\tau=t+1}^{n} \mathbb{1}\left\{a_{\tau}=C\right\} \mid \tilde{\xi}=\tilde{\xi}, Q(i)=t\right] \\
& =E_{\mu^{*}}\left[\sum_{\tau=1}^{t-1} \mathbb{1}\left\{a_{\tau}=C\right\} \mid \xi=\tilde{\xi}, Q(i)=t\right]+n-t
\end{aligned}
$$

Similarly,

$$
\begin{gathered}
E_{\mu^{D}}\left[G_{-i} \mid \xi=\tilde{\xi}, Q(i)=t\right]=E_{\mu^{D}}\left[\sum_{\tau=1}^{t-1} \mathbb{1}\left\{a_{\tau}=C\right\}+\sum_{\tau=t+1}^{n} \mathbb{1}\left\{a_{\tau}=C\right\} \mid \xi=\tilde{\xi}, Q(i)=t\right] \\
=E_{\mu^{*}}\left[\sum_{\tau=1}^{t-1} \mathbb{1}\left\{a_{\tau}=C\right\} \mid \tilde{\xi}=\tilde{\xi}, Q(i)=t\right]+E_{\mu^{*}}\left[\sum_{\tau=t+1}^{n} \mathbb{1}\left\{a_{\tau}=C\right\} \mid a_{t}=D\right]
\end{gathered}
$$

So $v_{t}(\gamma)$ is given by

$$
v_{t}(\gamma)=n-t+1-E_{\mu^{*}}\left[\sum_{\tau=t+1}^{n} \mathbb{1}\left\{a_{\tau}=C\right\} \mid a_{t}=D\right]
$$

To solve explicitly for $v_{t}(\gamma)$, note that $v_{n+1}=n-n+1=1$ and $v_{t}=1+(1-\gamma) v_{t+1}$. Solving for this first order linear difference equation leads directly to the expression for $v_{t}(\gamma)$ in Lemma 7. To see that $v_{t}(\gamma)$ is continuous also at $\gamma=0$, apply L'Hôpital's rule. It is easy to show that for all $\gamma<1, v_{t}(\gamma)>v_{t+1}(\gamma)$. To see that $\partial v_{t}(\gamma) / \partial \gamma<0$, note that $\partial v_{t}(\gamma) / \partial \gamma=\gamma^{-2}\left[(1-\gamma)^{n-t}[1+\gamma(n-t)]-1\right]$. We need to show that $(1-$ $\gamma)^{n-t}[1+\gamma(n-t)]<1$ for all $\gamma \in(0,1)$ and for all $t<n$. This is equivalent to showing $(1-\gamma)^{t}[1+t \gamma]<1$ for all $t \geq 1$ and for all $\gamma \in(0,1)$. We do this by induction. Note that 
for $t=1,(1-\gamma)(1+\gamma)<1$. Assume next that this is true for some $t$. Then

$$
(1-\gamma)^{t}[1+t \gamma]<1 \Leftrightarrow(1-\gamma)^{t+1}[1+(t+1) \gamma]<1-\gamma\left(1-(1-\gamma)^{t+1}\right)<1 .
$$

Let us turn next to $f_{t}(\gamma)$ :

$$
f_{t}(\gamma)=\operatorname{Pr}[Q(i)=t \mid \xi=(1,0)]=\frac{\sum_{\tau=1}^{t-1}(1-\gamma)^{t-\tau-1}}{\sum_{t=2}^{n} \sum_{\tau=1}^{t-1}(1-\gamma)^{t-\tau-1}}
$$

But $\sum_{\tau=1}^{t-1}(1-\gamma)^{t-\tau-1}=\gamma^{-1}\left[1-(1-\gamma)^{t-1}\right]$. This leads directly to the expression for $f_{t}(\gamma)$ in Lemma 7. To see that $f_{t}(\gamma)$ is continuous also at $\gamma=0$, apply L'Hôpital's rule. It is easy to show that for all $\gamma<1, f_{t}(\gamma)<f_{t+1}(\gamma)$.

\section{A.3 Proof of Lemma 3}

Assume that there exists an equilibrium profile $\sigma$ that features a positive level of contribution on the equilibrium path. Take any agent $i \in \mathcal{I}$ who contributes with positive probability: $\sigma_{i}(C \mid \xi)>0$ for some sample $\xi \in \Xi$. Let $\mu$ be beliefs consistent with $\sigma$ and $\tilde{\mu}$ the beliefs on future events if instead agent $i$ defects. Then,

$$
\begin{aligned}
& E_{\mu}\left[u_{i}\left(a_{i}, G_{-i}\right) \mid \xi\right] \geq E_{\tilde{\mu}}\left[u_{i}\left(a_{i}, G_{-i}\right) \mid \xi\right] \\
& E_{\mu}\left[r / n\left(G_{-i}+1\right)-1 \mid \xi\right] \geq E_{\tilde{\mu}}\left[r / n G_{-i} \mid \xi\right] \\
& E_{\mu}\left[\frac{r}{n}\left(\sum_{\tau=1}^{Q(i)-1} \mathbb{1}\left\{a_{\tau}=C\right\}+\sum_{\tau=Q(i)+1}^{n} \mathbb{1}\left\{a_{\tau}=C\right\}+1\right)-1 \mid \xi\right] \geq \\
& E_{\tilde{\mu}}\left[\frac{r}{n}\left(\sum_{\tau=1}^{Q(i)-1} \mathbb{1}\left\{a_{\tau}=C\right\}+\sum_{\tau=Q(i)+1}^{n} \mathbb{1}\left\{a_{\tau}=C\right\}\right) \mid \xi\right]
\end{aligned}
$$




$$
\begin{aligned}
E_{\mu} & {\left[\frac{r}{n} \sum_{\tau \geq Q(i)} \mathbb{1}\left\{a_{\tau}=C\right\}-1 \mid a_{i}=C, \xi\right] \geq } \\
E_{\mu} & {\left[\frac{r}{n} \sum_{\tau \geq Q(i)} \mathbb{1}\left\{a_{\tau}=C\right\} \mid a_{i}=D, \xi\right] \geq 0 }
\end{aligned}
$$

Thus, a necessary condition for agent $i$ to contribute is that at least $n / r-1$ of her successors contribute:

$$
E_{\mu}\left[\sum_{\tau>Q(i)} \mathbb{1}\left\{a_{\tau}=C\right\} \mid a_{i}=C, \xi\right] \geq \frac{n}{r}-1
$$

Let us focus on agents placed in positions $m+1$ to $n$. The random variable Count ${ }_{i}$ only considers agents who are not in the first $m$ positions. It keeps track of the number of agents who contribute after agent $i$ does so:

$$
\text { Count }_{i} \equiv \mathbb{1}\{Q(i)>m\} \mathbb{1}\left\{a_{i}=C\right\} \sum_{\tau>Q(i)} \mathbb{1}\left\{a_{\tau}=C\right\}
$$

Let $\widetilde{G} \equiv \sum_{t=m+1}^{n} \mathbb{1}\left\{a_{t}=C\right\}$. Then Count $\equiv \sum_{i \in \mathcal{I}}$ Count $_{i}=\widetilde{G}(\widetilde{G}-1) / 2$. Let $\Xi^{F}$ denote the set of all the samples that contain $m$ actions.

$$
\begin{aligned}
E_{\mu}(\text { Count }) & \equiv E_{\mu}\left[\sum_{i \in \mathcal{I}}\left[\mathbb{1}\{Q(i)>m\} \mathbb{1}\left\{a_{i}=C\right\} \sum_{\tau>Q(i)} \mathbb{1}\left\{a_{\tau}=C\right\}\right]\right] \\
& =\sum_{i \in \mathcal{I}} E_{\mu}\left[\mathbb{1}\{Q(i)>m\} \mathbb{1}\left\{a_{i}=C\right\} \sum_{\tau>Q(i)} \mathbb{1}\left\{a_{\tau}=C\right\}\right] \\
& =\sum_{i \in \mathcal{I}} \sum_{\xi \in \Xi^{F}} \operatorname{Pr}(\xi) E_{\mu}\left[\mathbb{1}\left\{a_{i}=C\right\} \sum_{\tau>Q(i)} \mathbb{1}\left\{a_{\tau}=C\right\} \mid \xi\right] \\
& =\sum_{i \in \mathcal{I}} \sum_{\xi \in \Xi^{F}} \operatorname{Pr}(\xi) E_{\mu}\left[\sum_{\tau>Q(i)} \mathbb{1}\left\{a_{\tau}=C\right\} \mid a_{i}=C, \xi\right] \sigma_{i}(C \mid \xi)
\end{aligned}
$$


Then by equation (4):

$$
\begin{aligned}
E_{\mu}(\text { Count }) & \geq \sum_{i \in \mathcal{I}} \sum_{\xi \in \Xi^{F}} \operatorname{Pr}(\xi)(n / r-1) \sigma_{i}(C \mid \xi) \\
& =(n / r-1) \sum_{i \in \mathcal{I}} E\left[a_{i} \mid Q(i)>m\right] \operatorname{Pr}(Q(i)>m)=E_{\mu}[(n / r-1) \widetilde{G}]
\end{aligned}
$$

But the number of successors who contribute is $E_{\mu}($ Count $)=E_{\mu}[\widetilde{G}(\widetilde{G}-1) / 2]$. Then,

$$
E_{\mu}\left[\left(\frac{n}{r}-1\right) \widetilde{G}\right]>E_{\mu}\left[\frac{n-1-m}{2} \widetilde{G}\right] \geq E_{\mu}\left[\frac{\widetilde{G}-1}{2} \widetilde{G}\right]=E_{\mu}(\text { Count }) \geq E_{\mu}\left[\left(\frac{n}{r}-1\right) \widetilde{G}\right]
$$

The first (strict) inequality comes from $r<2[1+(m-1) /(n-m+1)]$. The second (weak) inequality follows from the fact that $\widetilde{G} \leq n-m$.

This contradiction shows that nobody contributes after observing a full sample of size $m$. By backward induction, no one in the first $m$ positions contributes either. Therefore, in equilibrium there is no contribution if $r<2[1+(m-1) /(n-m+1)]$.

\section{A.4 Proof of Lemma 4}

Following a similar argument to the one in Lemma 1, an agent who observes defection also defects. Therefore, the signal $S_{Q(i)}$ may only matter when the agent receives a sample $\xi \in \Xi^{C}$. In two distinct cases the information contained in $S_{Q(i)}$ plays no role. First, if $\xi=\left(\xi^{\prime}, \xi^{\prime \prime}\right)$ has $\xi^{\prime}<m$, the agent learns her position immediately: she is in position $\xi^{\prime}+1$. Then, the signal is uninformative. Second, if $\xi=\left(\xi^{\prime}, \xi^{\prime \prime}\right)$ is such that $\xi^{\prime}=m$, the agent understands that she is not in any of the first $m$ positions. So she disregards any 
signal $S_{Q(i)} \in\{1, \ldots, m\}$.

Consider an agent who observes a sample of size $m$, where all agents cooperate: $\xi=$ $(m, m) \equiv \xi^{f u l l}$. The agent has the strongest incentives to defect when $S_{Q(i)}=n$. In what follows, we derive the condition that guarantees contribution after receiving such a signal. The same condition then guarantees contribution for any other signal. Let $\lambda^{\prime} \equiv$ $\operatorname{Pr}\left(Q(i)=n \mid s=n, \xi=\xi^{f u l l}\right)=\lambda /[1-m(1-\lambda) /(n-1)]$.

An agent who receives signal $s=n$ and sample $\xi=\xi^{f u l l}$ contributes whenever:

$$
\begin{aligned}
E_{\mu^{*}}\left[u\left(a_{i}, G_{-i}\right) \mid s=n, \xi=\xi^{f u l l}\right] & \geq E_{\tilde{\mu}}\left[u\left(a_{i}, G_{-i}\right) \mid s=n, \xi=\xi^{f u l l}\right] \\
r-1 & \geq \sum_{t=m+1}^{n-1} \frac{r}{n}(t-1) \frac{1-\lambda^{\prime}}{n-m-1}+\lambda^{\prime} \frac{r}{n}(n-1)
\end{aligned}
$$

Substituting for $\lambda^{\prime}$ leads to:

$$
r \geq 2 n\left[2+\frac{(n-1-m)(n-m)}{(n-1) /(1-\lambda)-m}\right]^{-1}
$$

\section{A.5 Proof of Lemma 5}

Consider first $\xi \notin \Xi^{C}$. As shown in Lemma 1, an agent who observes defection never believes that she can prevent further defection. So she herself defects. Consider next (full) samples without defection: $\xi \in \Xi^{C}$. Following similar steps as in Appendix A.1, an agent cooperates whenever:

$$
E_{\mu^{*}}\left[u_{i}\left(a_{i}, G_{-i}\right) \mid \tilde{\xi}\right] \geq E_{\tilde{\mu}}\left[u_{i}\left(a_{i}, G_{-i}\right) \mid \tilde{\xi}\right]
$$




$$
n-1 \geq \frac{1}{n-m} \sum_{t=m+1}^{n}(1+g)(t-1)=\frac{1+g}{2}(n+m-1)
$$

Then, an agent who observes a full sample of cooperation cooperates whenever $g \leq 1-$ $2 m /(n+m-1)$. If instead the agent observes a sample of cooperation with $\xi^{\prime}<m$ actions, her incentives to cooperate are even stronger. 